\title{
Load Forecasting of Power System Based on Integrated Sample System and Cloud Computing
}

\author{
Wang Huizhong ${ }^{1, a}$, Liu Ke $\mathrm{Ke}^{2, \mathrm{~b}}$, Zhu Hongyi ${ }^{3, \mathrm{c}}$ \\ ${ }^{1}$ Lanzhou University of Technology, Lanzhou 730050, Gansu province, China \\ ${ }^{2}$ Lanzhou University of Technology, Lanzhou 730050, Gansu province, China \\ ${ }^{3}$ State Grid Gansu Electric Power Research Institute, Lanzhou 730050, Gansu province, China \\ a38878809@qq.com, b415797893@qq.com, ${ }^{\text {c3 } 376260555 @ q q . c o m ~}$
}

Keywords: Integrated Sample System. Cloud computing. Particle swarm optimization. LSSVM.

Abstract. According to the characteristics of the short-term load forecasting, this paper established a integrated sample system. Through the analysis of various factors and load data to evaluate the effects of various factors on load forecasting, choosing the most appropriate forecast samples. PSO-LSSVM-Cloud model is established using Cloud computing technology to improve the efficiency of prediction. Finally, the actual data to establish PSO-LSSVM-Cloud model simulation comparison. Experimental results show that this load forecasting method has high forecasting precision and efficiency.

\section{Introduction}

With the construction of the smart grid and marketization increasingly deepening, not only the safe and stable operation of power grid need higher requirements, the speed and acuracy of the short-term power load forecasting also put forward higher requirements[1]. Least square support vector machine (LSSVM) is relatively mature modela and has a wide range of applications in the load forecasting. In order to improve the accuracy of prediction, a lot of literature introduce the particle swarm algorithm[2], ant colony algorithm[3], bacterial colony chemotaxis[4] and other intelligent optimization algorithm into the parameters of LSSVM. These algorithms improve the prediction accuracy to some extent, but intelligent algorithm search for the optimal solution need by a large number of iterations and searching for the optimal objective function value, it can result in occupying the vast computing resources and reducing the prediction speed [5].

This article will be Combined extended short-term load forecasting methods[6] and conventional forecasting methods. Combination forecasting method improving the prediction precision at the same time also increased the amount of calculation, its large training samples and increase the training time greatly. In order to improve the prediction speed, cloud computing has been introduced into the load forecasting. The article uses the sample that selected by Integrated Sample System to improve the prediction accuracy of load forecasting, using cloud computing technology at the same time, in order to improve the prediction speed it need to set up PSO-LSSVM-Clould model on Windows Azure platform, finally using the real power load data for example analysis.

\section{Cloud computing}

Cloud computing is a kind of super computing model based on Internet, it dstributes computational task across a large number of distributed computers, in a remote data center, tens of thousands or tens of millions of computers will work together[7]. As a result, cloud computing can offer users a experience that even more than 10 trillion times per second of computing power.

Microsoft Windows Azure platform is one of the most useful Platform as a Service(PaaS). PaaS provide a platform for user to deploy own application. The user does not need to manage and control the cloud infrastructure, platform can provide the operating system and runtime code. The user can according to the specification of the platform and the language supported to write your own applications. The load forecasting program will deployment on Windows Azure, after the project is 
published on the cloud, you can use any networked device to land, to upload data and do load calculation anywhere, it is highly advantageous to the isolated power grid. At the same time using the huge computing power of cloud computing technology to operate, have reached to enhance the efficiency of forecast. Windows Azure platform is shown in fig.1.

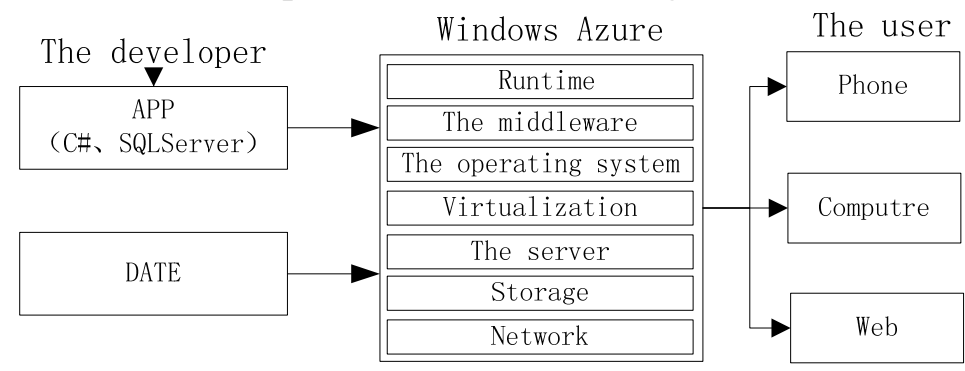

Fig.1. Windows Azure platform

\section{The Establishment of Integrated Sample System.}

The factors influencing power load is mainly the natural and social factors, this article collects historical load data and historical weather data as much as possible, Correlation analysis of these data, choosing one of the biggest factors that impact on load.

\section{The Choice of The Sample}

Meteorological factors is the largest impact for load. With the deepening of the research, the comprehensive meteorological factors have been proposed. Comprehensive meteorological factors considering the coupling effect of various meteorological factors. In this paper, considering the single meteorological factor is: temperature, precipitation, wind speed, relative humidity and so on. Comprehensive meteorological factors mainly are effective temperature, temperature humidity index and human comfort. Due to the regional climate of seasonal difference various meteorological factors are not necessarily suitable for becoming a training sample. Choose the most beneficial to load forecasting meteorological factors by correlation analysis.

The dimensions of the training sample is not the more the better, sometimes the more sample forecasting accuracy is lower. The first analysis of the local climate, then according to local climate and season features select a suitable meteorological factors as the training sample.

\section{The Establishment of Integrated Sample System}

In order to improve the efficiency of prediction, create a integrated sample system with impact load factors. For the selection of the sample should follow certain principles, not repeat, have layers, can complement each other. This paper collected a variety of indicators, the introduction of the extended short-term load forecasting. Integrated sample system is shown in fig. 2.

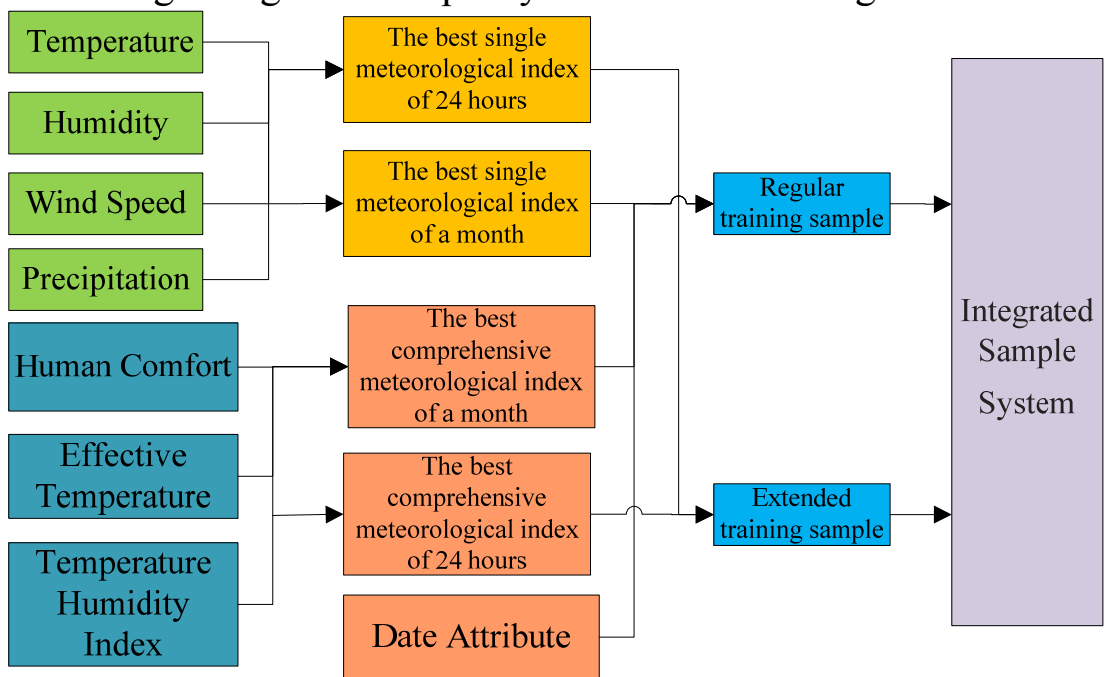

Fig.2. Integrated Sample System 
The figure 2 shows that the system collected single weather data and comprehensive meteorological data, four best sample is obtained by correlation analysis. Combining the four best sample with date properties to get the expanded training sample and the regular training sample, These two samples eventually make up integrated sample system.

\section{PSO-LSSVM-Cloud Model}

\section{Particle Swarm Optimization LSSVM}

Particle swarm optimization LSSVM (PSO-LSSVM) is an excellent performance of intelligent forecasting algorithms, simple and has a strong ability of rapid global optimization. This paper uses it to search the optimal kernel function parameters $\sigma$ and penalty parameter C of LSSVM.

PSO algorithm initialize a group of random particles, and then find the optimal solution through iteration. In each iteration the particles update themselves by tracking two extreme value. One is the particles themselves to find the optimal solution, namely the individual extreme value; The other one is the optimal solution searched by entire population, called the global solution[14]. Then particles according to two basic formula to update their speed and position. In a $\mathrm{N} d$ search space, $\mathrm{m}$ particles in a group, make the $\mathrm{Xi}=\left(\mathrm{x}_{\mathrm{i} 1}, \mathrm{x}_{\mathrm{i} 2}, \ldots, \mathrm{x}_{\mathrm{in}}\right)(\mathrm{i}=1,2, \ldots, \mathrm{m})$ is the location of $\mathrm{i}$ particle; $\mathrm{V}_{\mathrm{i}}=\left(\mathrm{v}_{\mathrm{i} 1}, \mathrm{v}_{\mathrm{i} 2}, \ldots, \mathrm{v}_{\text {in }}\right)$ is the rate of the i particle; $\mathrm{P}_{\text {ibest }}=\left(\mathrm{P}_{\mathrm{i} 1}, \mathrm{P}_{\mathrm{i} 2}, \ldots, \mathrm{P}_{\mathrm{in}}\right)$ is the optimal location of $\mathrm{i}$ particle, $\mathrm{P}_{\text {gbest }}=\left(\mathrm{P}_{\mathrm{g} 1}, \mathrm{P}_{\mathrm{g} 2}, \ldots, \mathrm{P}_{\mathrm{gn}}\right)$ is the optimal position of all particles. Follow these two optimal value, particles update their speed and position until meet the terminating condition.

\section{PSO-LSSVM-Cloud Model}

PSO-LSSVM algorithm using a large number of iterations and search for the optimal objective function value to search for the optimal solution, occupy the vast computing resources to reduce the speed prediction. This paper establishes a prediction model based on PSO-LSSVM algorithm and Cloud computing. The comprehensive sample system is divided into two cloud, respectively for regular load forecasting and extended short-term load forecasting, Finally, the prediction results are weighted to calculate the final prediction results. Model is shown in Fig. 3.

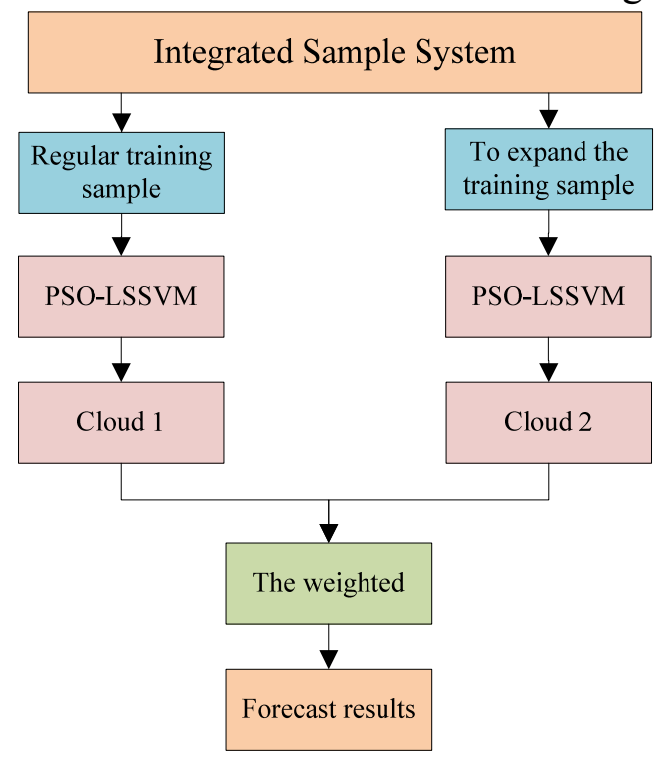

Weight formula is,

Fig.3. PSO-LSSVM-Cloud model

$$
\left\{\begin{array}{c}
\min _{w^{(j)}} e=\sum_{i=1}^{n} \sum_{t=1}^{T}\left(l_{i t}-\sum_{j=1}^{q} w^{(j)} \hat{l}_{i t}^{(j)}\right)^{2} \\
\text { s.t. } \quad \sum_{j=1}^{q} w^{(j)}=1 \\
w^{(j)} \geq 0
\end{array}\right.
$$


Where $w^{(j)}$ is the $g$ child node weights. $l_{i t}$ is the ith day load value at $\mathrm{t}$ moment, $\hat{l}_{i t}^{(j)}$ is the $\mathrm{j}$ child node virtual prediction results in the ith day at $t$ moments. By solving the minimization problem can get a set of weights, the ultimate load forecasting results is,

$$
\hat{l}_{t}=\sum_{j=1}^{q}\left(w^{(j)} \hat{l}_{t}^{(j)}\right)
$$

Where $\hat{l}_{t}$ is predicted load forecasting result at $\mathrm{t}$ moment.

\section{Simulation Research}

\section{The implementation of cloud computing}

In Windows 7 environment, first write the PSO-LSSVM Web application in the Visual Studio, then transferred Web applications to Windows Azure projects. The project attribute Instance set to 6, VM Size set to Medium. Means the project deployment operation ability is equal to 6 sets of dual-core CPU 1.6GHz, 3.5GB RAM physical host combined computing power on Windows Azure platform. Finally the project published to the cloud. Affinity Group choose north China means that the virtual machine was built in a computing center in north China.

\section{The example analysis}

This article uses the load value, meteorological factors and date attribute as the input samples of the south city in July 2014. Through the integrated sample system selected samples using the PSO-LSSVM-Cloud model for load forecasting. Forecast on July 28 to 31 load values. In order to verify the model, this article has carried on the contrast experiment. Due to the limited space, only compare forecasts at 12:00 every day.

Tab.1. Comparison of predict results

\begin{tabular}{cccccccc}
\hline \multirow{2}{*}{ DATE } & $\begin{array}{c}\text { Load } \\
(M W)\end{array}$ & \multicolumn{2}{c}{ PSO-LSSVM-Cloud } & \multicolumn{2}{c}{$\begin{array}{c}\text { No Integrated } \\
\text { Sample System }\end{array}$} & $\begin{array}{c}\text { No Integrated } \\
\text { Sample System } \\
\text { and No PSO }\end{array}$ \\
\cline { 3 - 8 } & & Forecast & MAPE & Forecast MAPE & Forecast MAPE \\
\hline 28 & 1067.3 & 1062.7 & $0.43 \%$ & 1058.3 & $0.84 \%$ & 1050 & $1.62 \%$ \\
29 & 1091.2 & 1102.4 & $1.03 \%$ & 1106.3 & $1.38 \%$ & 1108.1 & $1.55 \%$ \\
30 & 1035.2 & 1029 & $0.60 \%$ & 1027.5 & $0.74 \%$ & 1020.9 & $1.38 \%$ \\
31 & 1147.3 & 1142.5 & $0.42 \%$ & 1136.2 & $0.97 \%$ & 1171.4 & $2.1 \%$ \\
\hline $\begin{array}{c}\text { The average } \\
\text { MAPE }\end{array}$ & \multicolumn{2}{c}{$0.62 \%$} & $0.98 \%$ & $1.66 \%$ \\
\hline
\end{tabular}

As can be seen from the Table 1, the method introduced in this paper has the smallest error is only $0.62 \%$. The method error is $1.66 \%$ which does not consider the integrated sample system and does not consider the PSO. This proved that the prediction model established in this paper can improve the accuracy of prediction greatly.

In order to improve the accuracy using PSO algorithm, at the same time increase the amount of calculation and running time. Use the cloud for this purpose. The running time of the two methods were compared. 


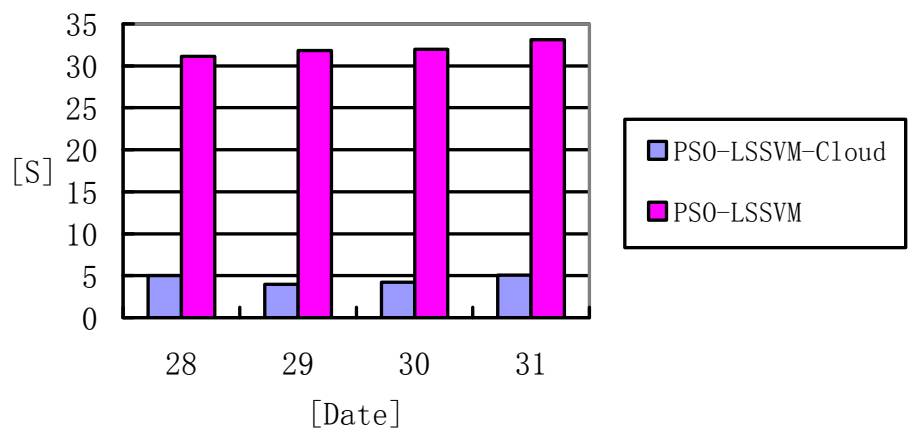

Fig.4. Model running time

As can be seen from the Fig.4. PSO-LSSVM method running time is about $33 \mathrm{~s}$, but the use of cloud computing running time is only 4 to 5 seconds. Thus, cloud computing reduces the operation time and improve the efficiency of prediction.

\section{Summary}

With the improvement of intelligent electricity network, power system tends to high-dimensional data. Load forecasting based on parameter optimization algorithm complex rate is higher and the stand-alone cannot afford such a large consumption of computing resources. In recent years the rise of cloud computing technology is one of the effective methods to solve large data operation. In this paper, based on comprehensive sample system and cloud computing power load forecasting algorithm, not only increase the accuracy of power load but also improve the efficiency of the operation greatly.

\section{Acknowledgements}

This work was financially supported by the Gansu Natural Science Foundation (1308RJZA117).

\section{References}

[1] Tang Conglan, Lu Jiping, Xie Yingzhao: Power System Technology. Vol. 38 (2014), p. 2014-2020.

[2] Shi Biao, Li Yu-xia, Yu Xin-hua, Yan Wang: Power System Technology. Vol. 33 (2009) p.180-184.

[3] Long Wen, Liang Ximing, LONG Zuqiang: Journal of Central South University, Vol. 42 (2011), p.3408-3414.

[4] ZENG Ming, LÜ Chunquan, TIAN Kuo: Proceedings of the CSEE. Vol. 31(2011), p.93-99.

[5] Wang Baoyi, Zhao Shuo, Zhang Shaomin: Power System Technology. Vol. 38 (2014). p.526-531.

[6] Zhang Fang-ming, Li Junzhou, MAO Yi. Vol. 37 (2009), p.69-73.

[7] He Yao, Wang Wen Qing, Xue Fei: Conputer Technology and Developent. Vol. 23(2013), p.69-72.

[8] Liang Jinjin, Wu De: Computer Engineering and Design. Vol. 35(2014), p.293-296. 